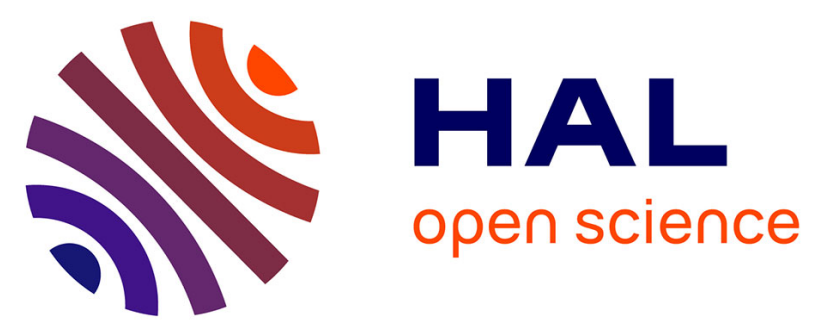

\title{
Hazardous Noxious Substance Detection Based on Hyperspectral Remote Sensing Technique
}

Jae-Jin Park, Kyung-Ae Park, Pierre-Yves Foucher, Philippe Deliot, Stephane Le Floch, Tae-Sung Kim, Sangwoo Oh, Moonjin Lee

\section{- To cite this version:}

Jae-Jin Park, Kyung-Ae Park, Pierre-Yves Foucher, Philippe Deliot, Stephane Le Floch, et al.. Hazardous Noxious Substance Detection Based on Hyperspectral Remote Sensing Technique. IGARSS 2020 - 2020 IEEE International Geoscience and Remote Sensing Symposium, Sep 2020, WAIKOLOA (virtual), United States. pp.2165-2168, 10.1109/IGARSS39084.2020.9324029 . hal-03224213

\section{HAL Id: hal-03224213 \\ https://hal.science/hal-03224213}

Submitted on 11 May 2021

HAL is a multi-disciplinary open access archive for the deposit and dissemination of scientific research documents, whether they are published or not. The documents may come from teaching and research institutions in France or abroad, or from public or private research centers.
L'archive ouverte pluridisciplinaire $\mathbf{H A L}$, est destinée au dépôt et à la diffusion de documents scientifiques de niveau recherche, publiés ou non, émanant des établissements d'enseignement et de recherche français ou étrangers, des laboratoires publics ou privés. 


\title{
HAZARDOUS NOXIOUS SUBSTANCE DETECTION BASED ON HYPERSPECTRAL REMOTE SENSING TECHNIQUE
}

\author{
Jae-Jin Park ${ }^{1)}$, Kyung-Ae Park ${ }^{2)}$, Pierre-Yves Foucher ${ }^{3)}$, Philippe Deliot ${ }^{3)}$, Stéphane Le Floch ${ }^{4)}$, \\ Tae-Sung Kim ${ }^{5)}$, Sangwoo Oh', Moonjin Lee ${ }^{5)}$ \\ ${ }^{1)}$ Department of Science Education, Seoul National University \\ ${ }^{2)}$ Department of Earth Science Education, Seoul National University \\ 3) Department of Theoretical and Applied Optics, Office National d'Etudes et Recherches Aérospatiales \\ ${ }^{4)}$ Service Recherche et Développement, Centre of Documentation, Research and Experimentation on \\ Accidental Water Pollution \\ ${ }^{5)}$ Maritime Safety Research Division, Korea Research Institute of Ships and Ocean engineering
}

\begin{abstract}
Hazardous Noxious Substance (HNS) is transported entirely through large vessels, so there is always a potential risk of marine HNS spills. In the event of an HNS accident, it can cause enormous human and property damage, so prompt detection is required. However, there is a limit to human access by ship, we need to use remote sensing data. In this study, ground experiments using hyperspectral cameras were performed to construct a spectral library of HNS. We classified the HNS and non-HNS by applying the hyperspectral mixture algorithm, and presented the HNS detection probability for every pixel by calculating the spectrum-based abundance fraction. The results of this study are expected to be used to estimate the extent of HNS spill in the event of a marine HNS accident.
\end{abstract}

Index Terms - Hazardous Noxious Substance (HNS), hyperspectral remote sensing, mixture, endmember, abundance fraction

\section{INTRODUCTION}

As the volume of marine transportation increases, ship accidents such as collisions and sinking have increased. The Korean Peninsula, which is surrounded by the sea on three sides, is more exposed to marine accidents due to its geographical characteristics. Marine accidents have also been increasing around the Korean peninsula over time. In particular, human-made disasters such as the Hebei Spirit oil spill accident in Taean in December 2007 and the oil spill in Yeosu in January 2014 caused considerable economic losses due to the leakage of large amounts of crude oil.

Recently, the risk of accidents on HNS as well as oil has been emphasized. In general, HNS is transported by large vessels at sea, so when a marine accident occurs, it causes not only spillage but also secondary damage due to fire and explosion risk. HNS also poses a risk of injury and disease due to spreading through the atmosphere or skin contact. In addition, it is very difficult to estimate the extent of marine damage because the marine accident site is not easily accessible to humans.

In the event of a marine HNS accident, it is possible to carry out on-site surveillance using a ship, but this may be limited in terms of space and time and may cause secondary damage. So, it is necessary to use remote sensing, which enables real-time monitoring and high-resolution view of the wide area. Satellite and airborne oil and HNS detection is an important topic in remote sensing fields.

Satellite sensors can be classified into multispectral sensors using only 5 to 6 channels of spectral information and hyperspectral sensors using wavelength bands divided into hundreds of narrow and continuous channels. In general, hyperspectral sensors are more effective for oil and HNS detection because they can take advantage of the reflective properties of objects in various channels.

Since HNS contains about 6,000 species and each substance is different in nature and has a high risk, it is difficult to perform the actual experiment. But we conducted ground experiments with HNS including toluene and xylene using hyperspectral sensors in the collaboration with ONERA and CEDRE organizations. In this study, three objectives were presented through HNS experiments. First, HNS spectrum was constructed by performing HNS measurement. Second, we developed a hyperspectral mixture analysis suitable for detecting HNS. Third, HNS and water classification methods are presented as probabilities based on the abundance fraction of unit pixels.

\section{EXPERIMENT}

HNS ground experiments were conducted at the CEDRE laboratory in BREST, France. The HNS used in the experiment was mainly selected from suspended HNS with 
high frequency of spill and included toluene, xylene, styrene, acetone and acetate vinyl (Fig. 1a). The period of the experiment is from September 2 to 7, 2019, depending on the weather conditions. The outdoor pool has a length of $20 \mathrm{~m}$, a width of $11 \mathrm{~m}$ and a depth of $2 \mathrm{~m}$ (Fig. 1b). In this experiment, a frame with a length of $4 \mathrm{~m}$ and a width of $2 \mathrm{~m}$ was fabricated, mainly wood or aluminum, and HNS was leaked only inside the frame (Fig. 2a, b). The edges of the frame were connected to the ground using a rope to fix the frame.

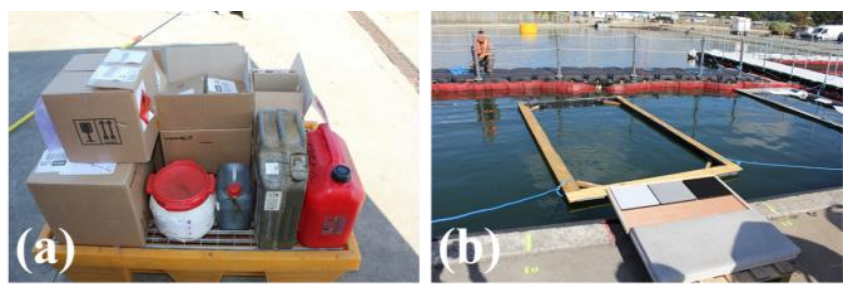

Fig. 1. (a) HNS used in ground experiment and (b) frame located inside marine pool.
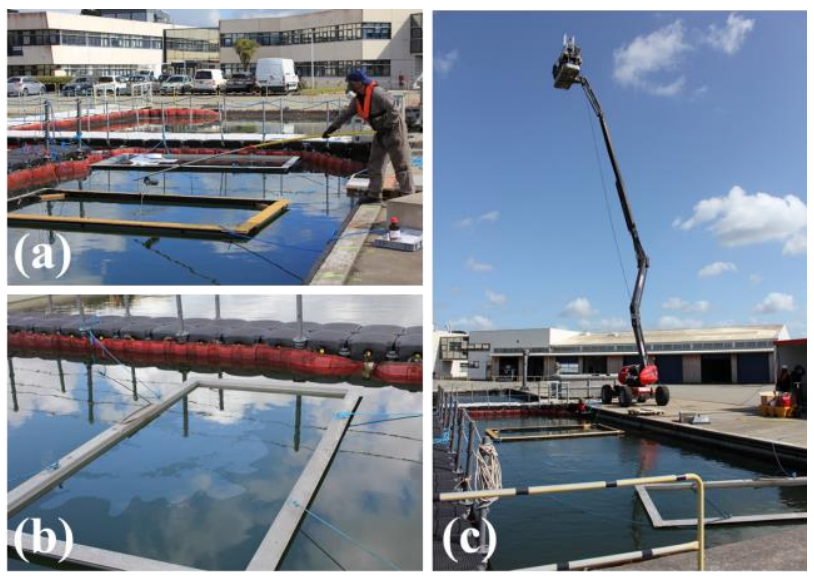

Fig. 2. (a) Process of spilling $1 \mathrm{~L}$ of toluene in the center of frame and (b) Spatial distribution of 1L of toluene spilled inside the frame. (c) hyperspectral camera located at $10 \mathrm{~m}$ height using lift.

The HNS was placed in the Erlenmeyer flask, and the long pole was used to drain the HNS in the center of the frame. The hyperspectral sensor was used by bonding two cameras, the VNIR-1600 and SWIR-1800 manufactured by HySpex. This means that HNS spectral characteristics can be analyzed for every channel from visible to SWIR wavelength band, that is, $400-2500 \mathrm{~nm}$. A hyperspectral camera was installed on a lift about 10 meters high to measure a high-resolution image of about $0.5 \mathrm{~cm}$, including a frame (Fig. 2c). In order to compare the spectral characteristics according to the volume of $\mathrm{HNS}, 1 \mathrm{~L}$ and $2 \mathrm{~L}$ were discharged, respectively.

\section{METHODS}

\subsection{Preprocessing of the HNS hyperspectral image}

Principal Component Analysis (PCA) was applied as a preprocessing method for HNS detection in hyperspectral images [1]. Hyperspectral image contains a large amount of spectral information, a dimensional compression procedure is required for the extraction of feature information. PCA is one of the most representative methods for the dimension compression of hyperspectral data, which is related to the noise reduction of an image and the compression using orthogonal function transformation (Fig. 3a). This method is a system that linearly converts the data into a new coordinate system by projecting the data on one axis. In this case, the axis with the largest variance is set as the first principal component and the axis with the second variance is set as the second principal component.

(a)

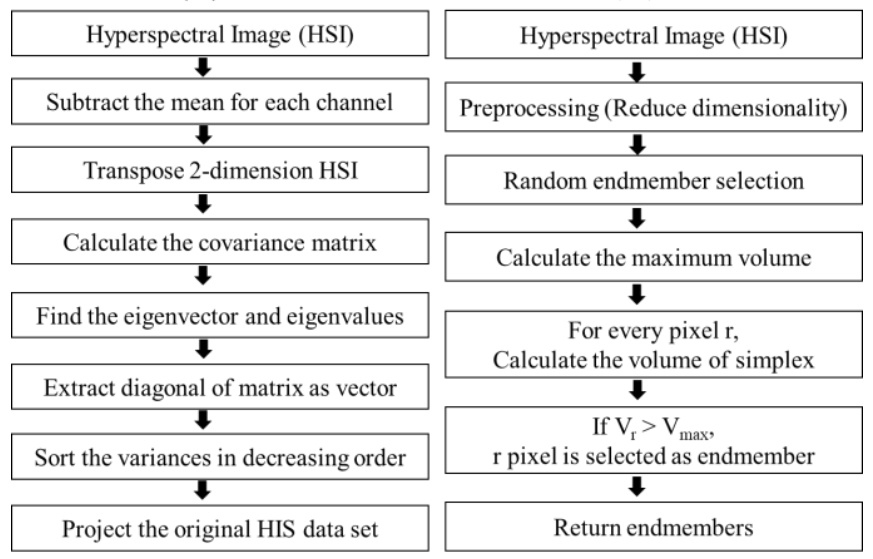

Fig. 3. Flow chart of the hyperspectral image data analysis for HNS detection using the (a) Principal Component Analysis (PCA) and the (b) N-Findr hyperspectral mixture algorithm.

\subsection{Application of the N-Findr algorithm}

The spectrum of a unit pixel in the hyperspectral image including HNS consists of a linear combination of each endmember spectrum. Spectral mixture analysis is a technique that analyzes multiple spectra within a pixel by separating the abundance fractions of the various endmembers. The N-Findr is mainly used for spectral unmixing (Fig. 3b). It is assumed that the volume of the simplex composed of endmembers in an image is the largest [3]. The $p$ endmembers are extracted by training the combination of arbitrarily set $p$ pixels. The number of $p$ endmembers is selected in advance and the pixel combination is composed of images compressed in $p-1$ dimension. This has the advantage no input parameters other than the number of pure substances is required. The endmember refers to an unmixed independent pixel such as HNS or water in the hyperspectral image. 


\section{RESULTS}

\subsection{Comparison of HNS Spectrum}

We analyzed the HNS spectral trend by comparing the ratio between toluene, xylene, styrene, acetone, acetate vinyl, condensate, gas oil and water (Fig. 4). Toluene shows a trend similar to water in the visible wavelength between 450 and $700 \mathrm{~nm}$, but the radiance is increasing rapidly after 700 $\mathrm{nm}$. Xylene and styrene have increased in radiance since the wavelength bands of about $560 \mathrm{~nm}$ and $800 \mathrm{~nm}$, respectively. In contrast, acetone shows a spectral trend similar to water in the visible wavelength range. Acetate vinyl shows a peak at $750 \mathrm{~nm}$. Like xylene, Condensate has an increased radiance at $570 \mathrm{~nm}$ wavelength, and gas oils generally exhibit lower radiance than water above $600 \mathrm{~nm}$.
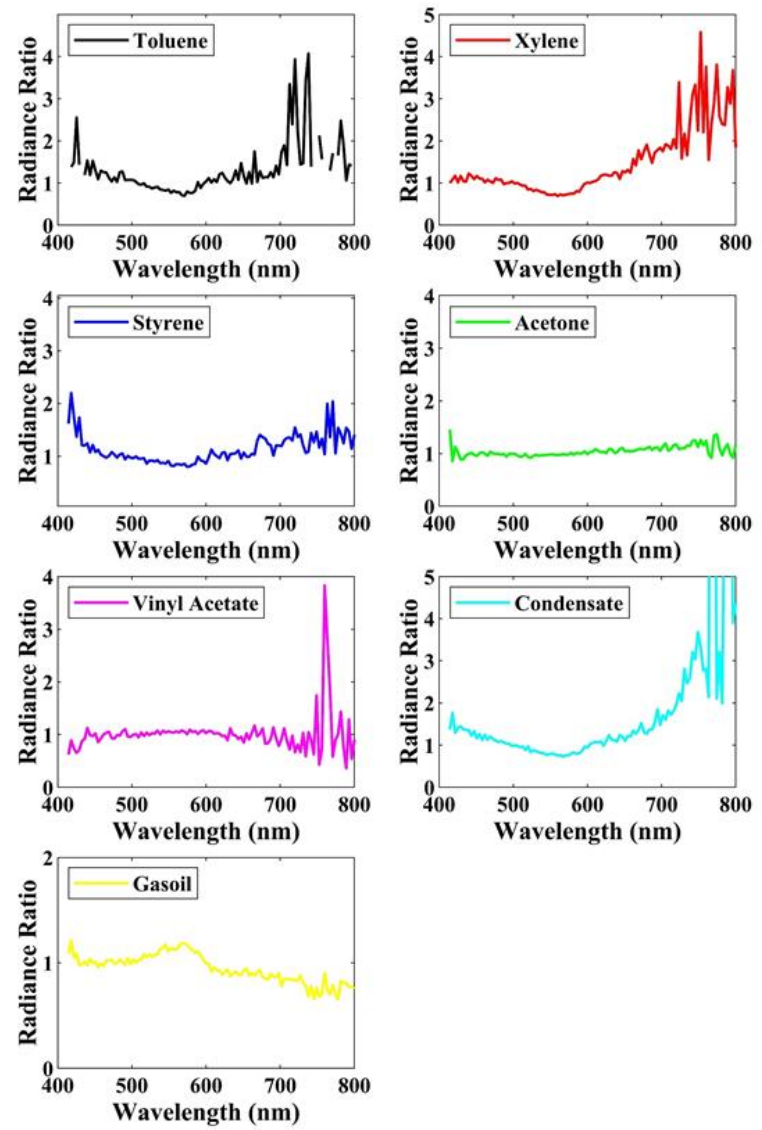

Fig. 4. The HNS spectral radiances at visible wavelength from ground experiments.

Most HNSs show higher radiance than water in the SWIR wavelength range, but acetone shows a spectral trend similar to water (Fig. 5). In particular, toluene and styrene show a high radiance of about 2.5 times, and gas oil shows a constant trend of about 2 times. Xylene and condensate show opposite trends at $1000-1500 \mathrm{~nm}$.

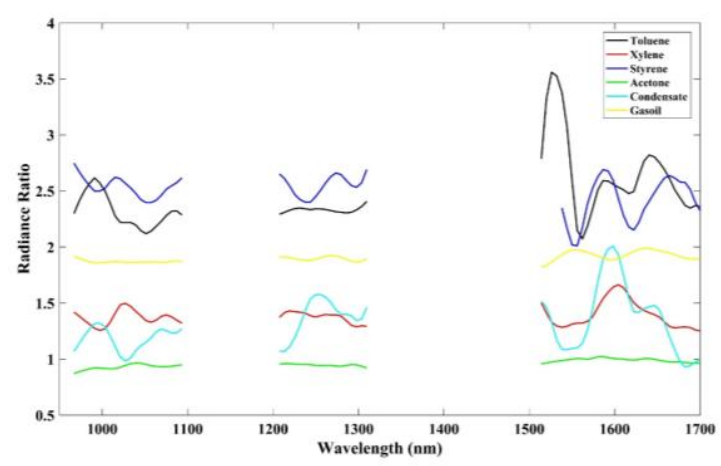

Fig. 5. The HNS spectral radiances at shortwave infrared (SWIR) wavelength from ground experiments.

\subsection{Extraction of endmember spectrum}

Fig. 6a shows the RGB composite image measured by the VNIR-1800 hyperspectral camera after 1L of toluene was leaked. We confirmed the presence of toluene in the frame by applying Gaussian fitting to the RGB image (Fig. 6b). N-Findr detection algorithm was applied to extract representative HNS and water spectrum in hyperspectral HNS images. Before applying the detection technique, we must select the number of endmembers. Two endmembers were selected because only toluene and water were detected and classified. The band was compressed using the PCA technique as a preprocess. And the spectrum corresponding to only two spectra (toluene \& water) occupying the maximum volume in the image was extracted by applying the N-Findr technique (Fig. 7).
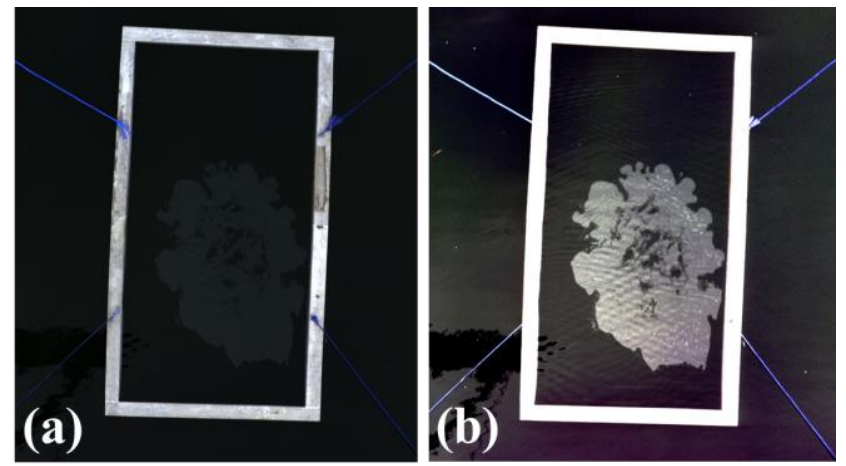

Fig. 6. (a) RGB composite images of hyperspectral image data using three bands, that is, red $(610.73 \mathrm{~nm})$, green $(559.84 \mathrm{~nm})$, and blue (454.42 nm) and (b) gaussian fitting image.

Endmember-1, which has relatively low radiance, is a spectrum corresponding to water. The maximum radiance appears at about $565 \mathrm{~nm}$, and the radiance is generally lowered toward the longer wavelength. Endmember-2, shown in red, is the spectrum corresponding to toluene. Toluene shows a double peak at $485 \mathrm{~nm}$ and $550 \mathrm{~nm}$, and, like water, shows a general decrease in long wavelengths. We found that toluene, unlike water, had a peak at $485 \mathrm{~nm}$ and showed about three times greater radiance than water. 


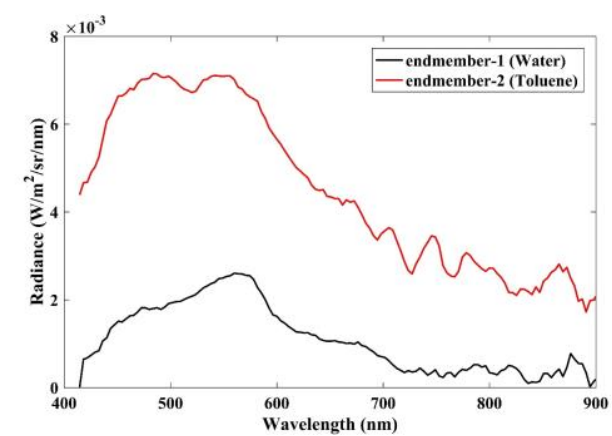

Fig. 7. The spectral radiances of (a) the two endmembers extracted from HNS hyperspectral image data by the $N$-Findr algorithm.

\subsection{HNS detection based on abundance fractions}

The spectrum of the unit pixel of the hyperspectral image is composed of a linear combination of spectra of given endmembers, the influence of each endmember can be represented by an abundance fraction map. The sum of the abundance fractions is $100 \%$. It is assumed that all the pixels of the aforementioned HNS hyperspectral image consist of a linear combination of toluene and water spectra. The endmember extracted by the N-Findr method satisfies the two constraints proposed by [3], that is, the Abundance Sum-to-one Constraint (ASC) and the Abundance Nonnegative Constraint (ANC).
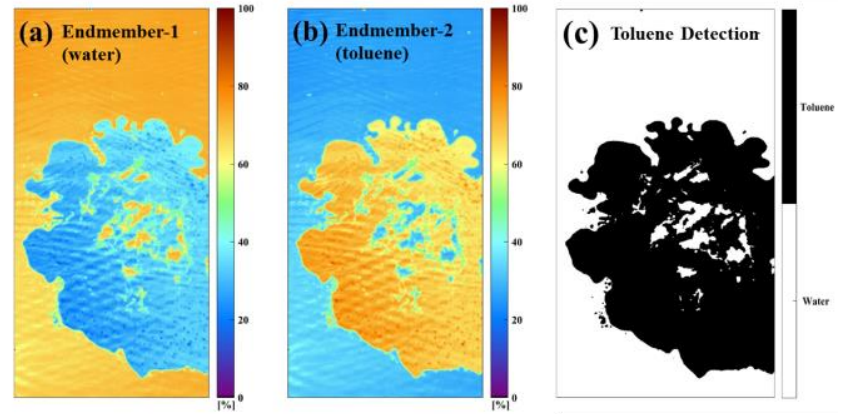

Fig. 8. Spatial distribution of the abundance fractions (\%) of (a) endmember-1 (water), (b) endmember-2 (toluene), and (c) result of the toluene detection based on endmember spectrum.

Figure 8a shows the abundance fraction corresponding to the water spectrum, which shows more than $80 \%$ of fraction at the top and bottom, but less than $40 \%$ at the center of the toluene distribution. On the contrary, the toluene abundance fraction is about $60 \%$ in the center and low radiance is less than about $20 \%$ in water. The hyperspectral image is composed of a mixture of toluene and water and shows a relatively different fraction according to the concentration. Assuming that toluene is a pixel in which toluene occupies more than half abundance fraction, it is possible to classify as shown in Figure 8c. In addition, the area occupying about $80 \%$ in Figure $8 \mathrm{~b}$ shows the concentration of the toluene spectrum in the form of dots. With optical sensors, it is impossible to detect HNS and determine the extent of spillage when a colorless HNS leak occurs. However, we can estimate the HNS spill area by applying the HNS hyperspectral mixture technique.

\section{CONCLUSION}

As the world seaborne trade on ships increases, there are frequent maritime accidents of oil and HNS spills. HNS spills on the coast cause not only economic damage but also environmental damage from marine ecosystem destruction. In this study, ground experiments were performed on toluene, xylene, styrene, acetone, acetate vinyl, condensate, and gas oil. HNS spectral libraries were constructed from visible to short-wave infrared wavelengths. We applied N-Findr, a hyperspectral mixed analysis technique, to detect HNS in the image based on the endmember spectrum. The relative ratio between HNS and water for all unit pixels is presented by calculating the abundance fraction of the HNS and water spectrum. The results of this study are meaningful in that it is possible to present the HNS spill area based on the spectrum when a colorless HNS outflow accident occurs at sea.

\section{REFERENCES}

[1] C. Rodarmel, and J. Shan, "Principal component analysis for hyperspectral image classification," Survey Land Inform. Sci., Vol. 62, no. 2, pp. 115-122, Jan. 2002.

[2] M.E. Winter, "A proof of the N-FINDR algorithm for the automated detection of endmembers in a hyperspectral image," in Proc. International Society for Optics and Photonics, Florida, USA, 2004, pp 31-41.

[3] D. C. Heinz, "Fully constrained least squares linear spectral mixture analysis method for material quantification in hyperspectral imagery," IEEE Trans. Geosci. Remote Sens., Vol. 39, no. 3, pp. 529-545, Mar. 2001. 